\title{
Live birth outcomes of vitrified embryos generated under growth hormone stimulation are improved for women categorized as poor-prognosis
}

\author{
Kevin N Keane ${ }^{1,2 *}$, Yun Ye ${ }^{1,3 *}$, Peter M Hinchliffe' ${ }^{1}$, Sheena LP Regan ${ }^{1,2,4}$, Satvinder S Dhaliwal' ${ }^{5}$, John L Yovich ${ }^{1,2}$ \\ 'PIVET Medical Center, Perth; ${ }^{2}$ Faculty of Health Sciences, School of Pharmacy and Biomedical Science, Curtin University, Perth, Australia; ${ }^{3} Z$ Zhongshan \\ People's Hospital, Zhongshan City, China; ${ }^{4}$ Stem Cell and Cancer Biology Laboratory, School of Pharmacy and Biomedical Sciences, Curtin Health \\ Innovation Research Institute (CHIRI) and ${ }^{5}$ School of Public Health, Curtin University, Perth, Australia
}

Objective: To determine the clinical pregnancy (CP) and live birth (LB) rates arising from frozen embryo transfers (FETs) that had been generated under the influence of in vitro fertilization (IVF) adjuvants given to women categorized as poor-prognosis.

Methods: A registered, single-center, retrospective study. A total of 1,119 patients with first FETs cycle include 310 patients with poor prognosis (109 treated with growth hormone [GH], (+)GH group vs. 201 treated with dehydroepiandrosterone, (-)GH group) and 809 patients with good prognosis (as control, (-)Adj (Good) group).

Results: The poor-prognosis women were significantly older, with a lower ovarian reserve than the (-)Adj (Good) group, and demonstrated lower chances of CP $(p<0.005)$ and LB $(p<0.005)$. After adjusting for confounders, the chances of both CP and LB in the (+)GH group were not significantly different from those in the (-)Adj (Good) group, indicating that the poor-prognosis patients given GH had similar outcomes to those with a good prognosis. Furthermore, the likelihood of LB was significantly higher for poor-prognosis women given GH than for those who did not receive $\mathrm{GH}(p<0.028)$. This was further confirmed in age-matched analyses.

Conclusion: The embryos cryopreserved from fresh IVF cycles in which adjuvant $\mathrm{GH}$ had been administered to women classified as poor-prognosis showed a significant 2.7-fold higher LB rate in subsequent FET cycles than a matched poor-prognosis group. The women with a poor prognosis who were treated with GH had LB outcomes equivalent to those with a good prognosis. We therefore postulate that GH improves some aspect of oocyte quality that confers improved competency for implantation.

Keywords: Adjuvants; Autologous; Dehydroepiandrosterone; Melatonin; Single embryo transfer; Vitrified embryos

Received: April 11, 2019· Revised: July 3, 2019· Accepted: July 19, 2019 Corresponding author: Kevin N Keane

PIVET Medical Center, 166-168 Cambridge St, Perth, Western Australia 6007, Australia

Tel:+61-8-9422-5400 Fax:+61-8-9382-4576 E-mail: kevin.keane@curtin.edu.au Co-Corresponding author: John L Yovich

PIVET Medical Center, 166-168 Cambridge St, Perth, Western Australia 6007, Australia

Tel: +61-8-9422-5400 Fax:+61-8-9382-4576 E-mail:jlyovich@pivet.com.au

*These authors contributed equally to this study.

**The project was funded internally under an existing long-term collaboration between PIVET Medical Center and Curtin University, which supports a Fellowship position for KNK and an adjunct fellowship for SLPR.

This is an Open Access article distributed under the terms of the Creative Commons Attribution Non-Commercial License (http://creativecommons.org/licenses/by-nc/4.0/) which permits unrestricted non-commercial use, distribution, and reproduction in any medium, provided the original work is properly cited.

\section{Introduction}

The biological and biochemical mechanisms by which growth hormone (GH) can lead to better clinical outcomes in assisted reproductive technology (ART) remain unclear, but are believed to be related to oocyte development and/or subsequent embryo quality, along with potential effects in the endometrium. Several clinical studies over the past decade have focused on the use of $\mathrm{GH}$ for women classified as poor-prognosis in ART programs [1-9] as a desperate means to establish a pregnancy in severely infertile patients. However, the data around any beneficial effect is perplexing, with many studies claiming positive effects and an almost equivalent number of other investigations reporting no significant effect. In 2019, the most pow- 
erful prospective, randomized, double-blind, placebo-controlled trial was published, but failed to confirm any benefit from adjuvant $\mathrm{GH}$ on clinical outcomes such as the clinical pregnancy (CP) and live birth (LB) rate [8]. However, this prospective randomized controlled trial (RCT) — known as the Live birth, In vitro fertilisation and Growth Hormone Treatment (LIGHT) study - failed to reach its enrolment target, as patients categorized as poor-prognosis resisted inclusion and only $34.9 \%$ of the required sample size was recruited $[8,10]$. Nonetheless, the report demonstrated that $\mathrm{GH}$ patients reached oocyte retrieval faster than non-GH patients, as also shown in other studies [9], which may indicate that $\mathrm{GH}$ has an effect on folliculogenesis; however, this possibility was not supported by differences in embryo quality [8]. Nonetheless, the links between $\mathrm{GH}$ and folliculogenesis, oocyte quality and responsiveness to gonadotrophins is still unclear $[2,9,11]$. The authors also indicated that while there was no difference in the number of oocytes retrieved or transferred, the low recruitment numbers may mask any perceived improvements [8].

It is increasingly difficult to recruit patients into prospective, welldesigned ART studies and to convince them to devote a portion of their narrow reproductive lifespan to research. The LIGHT study performed admirably given that it was conducted in a setting where patients could avail themselves of $\mathrm{GH}$ outside of the trial via the clinician and then not consent to study inclusion [8]. Consequently, the unique commercial availability of ART services means that patient demand overpowers the ability to recruit, leaving the field with vastly underpowered studies. This may mean that alternative, weaker studies, such as retrospective cohort studies, may be required to advance the field provided they are stringently designed, conducted and analysed. Two recent retrospective studies by our group showed that $\mathrm{CP}$ and $\mathrm{LB}$ rates were significantly greater with $\mathrm{GH}$ (3.42-fold and 6.16-fold higher, respectively) following the transfer of fresh autologous embryos in stimulated cycles $[12,13]$. Age at embryo transfer (ET) and morphological quality of the transferred embryo were the only other significant independent predictors of the likelihood of CP and $L B$, but no significant differences were observed in the proportion of high-quality embryos generated in the $\mathrm{GH}$ arm, echoing the most recent prospective study [8]. While these examinations $[12,13]$ were undertaken as retrospective, observational analyses and have significant limitations, they represent one of the largest current GH data-sets published. However, these reports focused on only fresh ET cycles and not subsequent frozen cycles, which are also lacking in the LIGHT study.

Some studies have already investigated the role of GH co-treatment directly with hormone replacement therapy (HRT) in frozen ET (FET) cycles, and indicated that $\mathrm{GH}$ may affect endometrium thickness, $\mathrm{CP}$ and LB rates [14-17]. Due to their study design, those investigations suggested that GH may have additonal positive effects on outcomes that are related to the endometrium, rather than embryo quality, as the embyros were generated previously without $\mathrm{GH}$ exposure. However, only one previous study has examined the cumulative effect of $\mathrm{GH}$ on outcomes in fresh and subsequent FET cycles, and demonstrated higher productivity (cumulative) in terms of $\mathrm{CP}$ and $\mathrm{LB}$ rates in GH-treated patients [1]. We have chosen to build upon this latter study and determine whether $\mathrm{GH}$ administration in the stimulation cycle can lead to a higher likelihood of CP and LB in subsequent FET HRT cycles in a new cohort of patients from our clinic. The current retrospective study may provide insight into any "carry-over" effect from $\mathrm{GH}$ administration during stimulation cycles into subsequent FET cycles, using a strict single ET and HRT regimen.

\section{Methods}

\section{Study cohort and definition of poor-prognosis patients}

This retrospective study was registered prior to commencement of the analysis (ACTRN12618001933246). All FET cycles initiated from 1 April, 2008 to 31 March, 2017 were extracted from our validated database $(n=2,857)$. The start date was selected as the time when embryo cryopreservation was almost exclusively performed using the Cryotop technique for vitrification and all current clinical and laboratory protocols were consistently applied [18]. Cycles in which the embryos were cryopreserved by the alternative slow-freeze method were excluded ( $n=339)$, leaving 2,518 cases for examination. The vast majority of FETs were performed using an HRT schedule $(n=2,208)$ and were the focus of this study, while FETs in natural cycles $(n=34)$ or using low-dose ovarian stimulation $(n=276)$ were excluded. Double ET was performed in 367 cases, which were also excluded from the analysis, leaving 1,841 single ET cycles. Finally, as a means to randomize and offset selection-bias, we focused only on the first chronological FET transfer cycle for each individual patient within the study period (i.e., not necessarily the very first in vitro fertilization [IVF]/FET naïve transfer), resulting in a final dataset consisting of 1,119 cycles/women, receiving a single, vitrified autologous embryo via ET.

In our practice, poor-prognosis women can receive GH or other IVF adjuvant (dehydroepiandrosterone [DHEA]) treatment in stimulation cycles if they have one of the following characteristics: age $\geq 40$ years, generating $\leq 3$ metaphase II oocytes despite high folliclestimulating hormone (FSH) stimulation doses ( $\geq 300 \mathrm{IU})$, $>50 \%$ poorquality embryos, repetitive implantation failure (RIF) in $\geq 3$ ETs without achieving pregnancy, and a low ovarian reserve (antral follicle count $[\mathrm{AFC}] \leq 8$ or anti-Müllerian hormone $[\mathrm{AMH}]$ level $\leq 8 \mathrm{pmol} / \mathrm{L}$ ), designated as AFC groups $D$ and $E$ in our published recombinant $F S H$ dosing algorithms $[19,20]$. These criteria have been published previously for fresh ET cycles, with group B/C corresponding to 9-19 folli- 
cles inclusive and group A equating to 20 or more follicles [12,13]. In the current FET study, patients receiving or having a medical history of receiving IVF adjuvants (GH or DHEA) were considered to have a poor prognosis for the above reasons. Consequently, the cohort was subdivided on the basis of adjuvant administration in any stimulation cycle from their entire medical history as an indication of a poor prognosis $(n=310)$. These patients were categorized further specifically on the basis of $\mathrm{GH}$ administration in the stimulation cycle that led to the analysed FET transfer cycle in the current study and designated as $(+) \mathrm{GH}(\mathrm{n}=109)$. If they did not receive $\mathrm{GH}$, but DHEA, then the cycles/patients were grouped into the $(-) \mathrm{GH}$ category $(n=201)$. This separation was appropriate since we have shown previously that DHEA did not significantly modulate outcomes versus GH [13]. To reiterate, both groups were considered to have a poor prognosis due to their medical history of using adjuvants in other cycles. Therefore, the control group comprised women who did not have a history of adjuvant usage at all, and were considered to have a good prognosis ([-]Adj [Good], $n=809)$.

\section{Clinical aspects of stimulation and FET/HRT treatment cycles}

Administration of $\mathrm{GH}$ to eligible patients occurred during the stimulation cycle. The aim was to administer 1-1.5 IU of GH per day. Six vials of Saizen (Merck Serono, Frenchs Forest, Australia) containing 9 IU of GH were given over 6 weeks in the lead-up to ovum pick-up, equating to $54 \mathrm{IU}$ over 33-37 days, with an average of approximately 1.5 IU per day. SciTropin (SciGen, Belrose, Australia) 0.3 mg was injected daily for 45 days prior to trigger, with patients receiving $\mathrm{GH}$ at precisely $1.0 \mathrm{IU}$ per day up to ovum pick-up. Saizen was used in 38 cases (34.9\%) and SciTropin in 71 cases (65.1\%). There was no significant difference in outcomes between both agents. Our FET HRT protocols have been described previously in detail [21]. Briefly, from day 1 to 10 patients received estradiol $\left(E_{2}\right)$ valerate (Progynova; Bayer, Reading, UK) tablets (4 mg three times per day) and endometrial thickness was graded on day 10 by a transvaginal ultrasound (TVUS) scan. Thereafter, $E_{2}$ pessaries were applied at a dosage (10 mg or 20 $\mathrm{mg}$ ) dependent on the endometrial characteristics. Progesterone (P4) pessaries were added when the endometrial thickness was $\geq 7.5$ $\mathrm{mm}$. The synchronous dating of ET placed a day 5 blastocyst into the uterus on P4 pessary day 6, while a day 3 cleavage-stage embryo was transferred on P4 pessary day 4. The luteal phase hormonal support comprised $4 \mathrm{mg}$ of oral Progynova with vaginal pessaries of 400 mg of P4 three times per day, and the evening P4 dose was combined with $2 \mathrm{mg}$ of $E_{2}$ ( $E_{2} / P 4$ combo). Midluteal $E_{2}$ and $P 4$ levels were measured, aiming for a value between 60 and $99 \mathrm{nmol} / \mathrm{L}$ and between 1,000 and 2,000 pmol/L for P4 and $E_{2}$, respectively [22]. Pessaries were adjusted to achieve these ranges. The regimen was continued throughout the first trimester of ensuing pregnancies, which were diagnosed by a beta-human chorionic gonadotropin level $>25$ IU at day 18-20 of P4 pessary introduction and categorized as "clinical" if an intrauterine gestational sac was shown at week 7 (i.e., 5 weeks after the commencement of $\mathrm{P} 4$ pessaries). Each birth was categorized as a LB if at least one infant was born alive.

\section{Embryo grading, handling and transfer}

Embryo quality was graded as high, mid-range and low for blastocysts according to our modification of the Gardner grading system $[23,24]$, and as high or low for cleavage-stage embryos as published previously [25]. All embryos were vitrified using the Cryotop protocol (Kitazato; Gytech, Hawthorn East, Australia) using HEPES-buffered medium containing the cryoprotectants $7.5 \%$ ethylene glycol (EG) and $7.5 \%$ dimethylsulphoxide (DMSO) for 15 minutes, followed by $15 \%$ EG and 15\% DMSO in $0.5 \mathrm{M}$ glucose for $50-60$ seconds. The embryos were then stirred through liquid $\mathrm{N}_{2}$ to vitrify and finally stored in liquid $\mathrm{N}_{2}$. For warming, all embryos were sequentially transferred through a thawing solution of $1.0 \mathrm{M}$ sucrose plus $20 \%$ human serum albumin (HSA), a diluting solution of $0.5 \mathrm{M}$ sucrose with 10\% HSA, and finally immersion into two washing solutions without sucrose, but with 10\% HSA. Then, they were placed into the appropriate cleavage- or blastocyst-stage culture medium and incubated prior to the ET procedure. Single ET was conducted under TVUS using a double catheter system (K-JETS-7019-SIVF; Cook Australia, Brisbane, Australia or Wallace Classic catheter, Gytech; Cooper Surgical, Sydney, Australia).

\section{Statistical analysis}

The normality of the data distribution was determined using the Shapiro-Wilk test. The means of normal data were compared using analysis of variance or the Student $t$-test as appropriate, while the non-parametric Kruskal-Wallis test was used to compare the medians of non-normally distributed data. Categorical variables were compared using the chi-square of ratios. Univariate and stepwise multi-variable binary logistic regression models were used to determine the influence of confounding variables in predicting the likelihood of CP and LB. These included female age at ET (i.e., cycle age), the age of the woman at embryo fertilization (i.e., embryo age), AFC, $\mathrm{AMH}$, body mass index (BMI), and transferred embryo stage and quality. The effect of each variable was expressed as an odds ratio $(\mathrm{OR})$, with a corresponding $95 \%$ confidence interval $(\mathrm{Cl})$. Due to the retrospective nature of this investigation, the adjuvant study groups were different in terms of number of patients and mean female age at ET. In order to analyze these groups in a format that allowed similarity in terms of these factors, we performed two separate cycle age-matched sub-analyses using the random case matching function of IBM SPSS ver. 24.0 (IBM Corp., Armonk, NY, USA) in a 1 to 1 ratio with an age tolerance of 1 year. The first sub-analysis compared 
2,518 All FET cycles

(April 1, 2008-March 31, 2017)

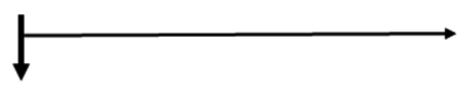

310 Excluded

276 Low-dose stimulation

34 Natural cycles

2,208 FET HRT cycles

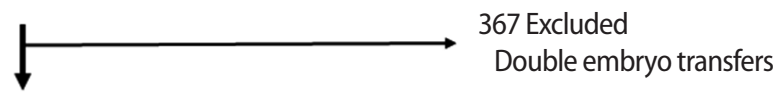

1,841 FET HRT with SET

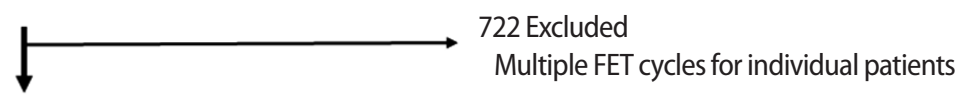

1,119 First cycle selected per patient

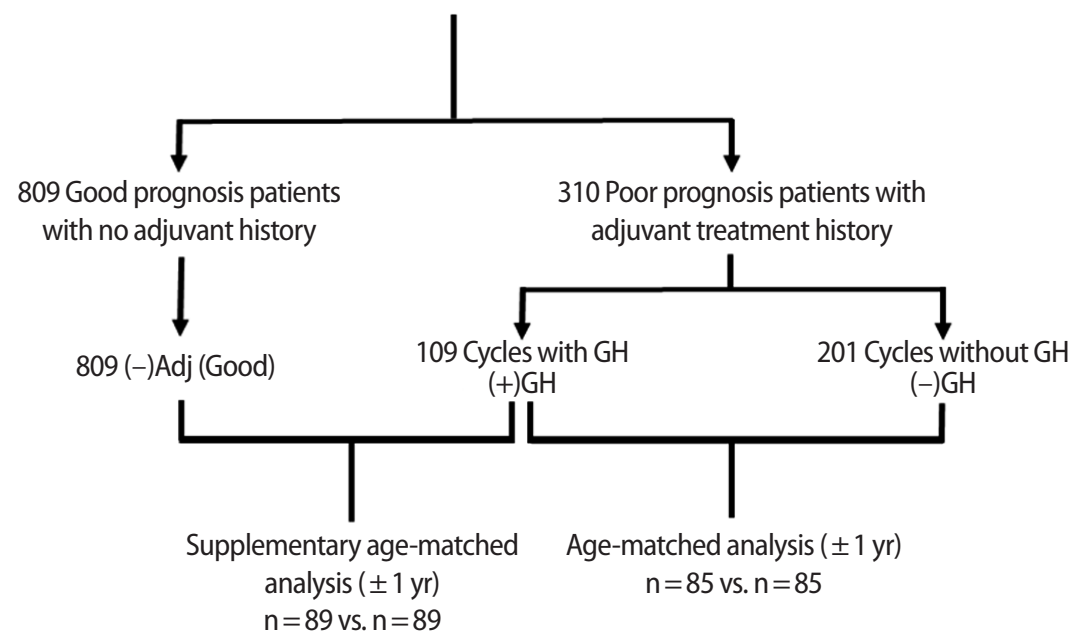

Figure 1. Flow diagram of data extraction and analysis. The date range was selected to ensure that embryos were cryopreserved using the same vitrification process. Natural and low-dose stimulation cycles were excluded to focus on cycles with hormone replacement therapy (HRT), while multiple embryo transfers were excluded to allow a focus on single embryo transfer (SET). Of the remaining cycles, only the first chronological cycle for each patient was included for analysis in an attempt to offset patient/cycle selection bias. Matched analyses of selected groups were performed using SPSS. FET, frozen embryo transfer; GH, growth hormone.

the (-)GH group to the $(+) \mathrm{GH}$ group and resulted in 85 exact-match cases. The second sub-analysis compared the (-)Adj (Good) group to the $(+) \mathrm{GH}$ group, and resulted in 89 exact-match cases (Figure 1).

\section{Compliance with ethical standards}

Our clinic is accredited with the national regulatory body, the Reproductive Technology Accreditation Committee, as well as the local Reproductive Technology Council of Western Australia. The retrospective analysis and reporting of anonymous data were approved under Curtin University Ethics Committee (approval no. RD_25-10). In addition, as part of our documentation system, written informed consent was obtained from each participant in accordance with the Declaration of Helsinki. Participants approved of the use of their anonymous data for research purposes, and also accepted the use of adjuvants, for which they were required to pay over and above the IVF treatment charges. There was no coercion of patients, who were all informed that repeated IVF treatments without adjuvants might be less expensive and equally effective.

\section{Results}

\section{Analysis of the whole cohort}

The adjuvant treatment groups were different with regard to the average female cycle and embryo age, and in the median serum AMH level (Table 1). No significant differences were found in the median BMI between the treatment groups. Overall, women who were considered to have a good prognosis ([-])Adj [good]), were younger and had a higher AMH level than those considered to have a poor 
Table 1. Overview of patient characteristics and clinical outcomes in the whole cohort

\begin{tabular}{|c|c|c|c|c|}
\hline \multirow{2}{*}{ Variable } & \multirow{2}{*}{ (-)Adj (Good) } & \multicolumn{2}{|c|}{ Poor-prognosis patients } & \multirow{2}{*}{$p$-value } \\
\hline & & $(-) \mathrm{GH}$ & $(+) \mathrm{GH}$ & \\
\hline No. of cycles & 809 & 201 & 109 & - \\
\hline Cycle age (yr) & $33.7 \pm 4.6$ & $36.5 \pm 4.3^{b)}$ & $39.4 \pm 4.7^{\mathrm{b}, \mathrm{c})}$ & $<0.005^{\mathrm{d})}$ \\
\hline Embryo age (yr) & $32.8 \pm 4.5$ & $35.8 \pm 4.4^{\mathrm{b})}$ & $38.8 \pm 4.8^{\mathrm{b}, \mathrm{c})}$ & $<0.005^{\mathrm{d})}$ \\
\hline $\mathrm{AMH}(\mathrm{pmol} / \mathrm{L})^{\mathrm{a})}$ & $18.8(29.1)$ & $9.2(18.6)^{b)}$ & $4.6(12.3)^{b)}$ & $<0.005^{\mathrm{e})}$ \\
\hline $\operatorname{BMI}\left(\mathrm{kg} / \mathrm{m}^{2}\right)^{\mathrm{a})}$ & $23.3(6.5)$ & $24.2(8.5)$ & $22.5(5.6)$ & $0.627^{e)}$ \\
\hline FET cycle & 809 & 201 & 109 & - \\
\hline FET pregnancy rate & $382 / 809(47.2)$ & $58 / 201(28.9)$ & $31 / 109(28.4)$ & $<0.005^{\mathrm{f})}$ \\
\hline FET live birth rate & $293 / 809(36.2)$ & $37 / 201(18.4)$ & $24 / 109(22.0)$ & $<0.005^{f)}$ \\
\hline FET miscarriage rate & 89/382 (23.3) & $21 / 58(36.2)$ & $7 / 31(22.6)$ & $0.101^{f)}$ \\
\hline
\end{tabular}

Values are presented as mean \pm standard deviation or number (\%) unless otherwise indicated.

GH, growth hormone; AMH, anti-Müllerian hormone; BMI, body mass index; FET, frozen embryo transfer.

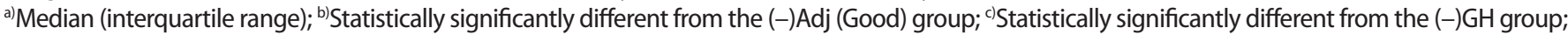

${ }^{\mathrm{d})}$ Analysis of variance; ${ }^{\mathrm{e}}$ Kruskal-Wallis test; ${ }^{\mathrm{f}}$ Chi-square test.

Table 2. Binary logistic regression analysis of the whole cohort

\begin{tabular}{|c|c|c|c|c|}
\hline \multirow{2}{*}{ Variable } & \multicolumn{2}{|c|}{ Clinical pregnancy likelihood } & \multicolumn{2}{|c|}{ Live birth likelihood } \\
\hline & Odds ratio $(95 \% \mathrm{Cl})$ & $p$-value & Odds ratio $(95 \% \mathrm{Cl})$ & $p$-value \\
\hline \multicolumn{5}{|l|}{ Univariate regression } \\
\hline \multicolumn{5}{|l|}{ Treatment type } \\
\hline (-)Adj (Good) & 1 & - & 1 & - \\
\hline$(-) \mathrm{GH}$ & $0.45(0.32-0.63)$ & $<0.005$ & $0.40(0.27-0.58)$ & $<0.005$ \\
\hline$(+) \mathrm{GH}$ & $0.44(0.29-0.69)$ & $<0.005$ & $0.50(0.31-0.80)$ & 0.004 \\
\hline Cycle age & $0.93(0.91-0.96)$ & $<0.005$ & $0.93(0.91-0.96)$ & $<0.005$ \\
\hline Embryo age & $0.93(0.91-0.96)$ & $<0.005$ & $0.93(0.91-0.96)$ & $<0.005$ \\
\hline Serum AMH & $1.01(1.01-1.02)$ & 0.001 & $1.01(1.00-1.01)$ & 0.02 \\
\hline BMI & $1.00(0.98-1.03)$ & 0.973 & $0.99(0.97-1.02)$ & 0.494 \\
\hline \multicolumn{5}{|l|}{ AFC group } \\
\hline A ( $\geq 20$ follicles) & 1 & - & 1 & - \\
\hline B/C (9-19 follicles) & $0.72(0.54-0.95)$ & 0.02 & $0.72(0.53-0.97)$ & 0.031 \\
\hline $\mathrm{D} / \mathrm{E}$ ( $\leq 8$ follicles $)$ & $0.64(0.47-0.87)$ & 0.004 & $0.69(0.50-0.95)$ & 0.022 \\
\hline \multicolumn{5}{|l|}{ Blastocyst versus cleavage } \\
\hline Cleavage (day 3) & 1 & - & 1 & - \\
\hline Blastocyst (day 5) & $2.58(1.72-3.87)$ & $<0.005$ & $2.33(1.49-3.64)$ & $<0.005$ \\
\hline \multicolumn{5}{|l|}{ Quality of transferred embryo } \\
\hline High-quality blastocyst (day 5) & 1 & - & 1 & - \\
\hline Medium/low-quality blastocyst (day 5) & $0.48(0.37-0.64)$ & $<0.005$ & $0.44(0.32-0.59)$ & $<0.005$ \\
\hline High-quality day 3 & $0.30(0.20-0.46)$ & $<0.005$ & $0.33(0.21-0.52)$ & $<0.005$ \\
\hline Low-quality day 3 & $0.14(0.07-0.27)$ & $<0.005$ & $0.17(0.08-0.36)$ & $<0.005$ \\
\hline \multicolumn{5}{|l|}{${\left.\text { Multivariate regression }{ }^{\mathrm{a}}\right)}$} \\
\hline \multicolumn{5}{|l|}{ Treatment type } \\
\hline (-)Adj (Good) & 1 & - & 1 & - \\
\hline$(-) \mathrm{GH}$ & $0.62(0.37-1.04)^{a)}$ & 0.067 & $0.38(0.21-0.70)^{a)}$ & 0.002 \\
\hline$(+) \mathrm{GH}$ & $0.78(0.40-1.54)^{a)}$ & 0.472 & $0.80(0.39-1.65)^{a)}$ & 0.551 \\
\hline
\end{tabular}

$\mathrm{Cl}$, confidence interval; $\mathrm{GH}$, growth hormone; $\mathrm{AMH}$, anti-Müllerian hormone; $\mathrm{BMI}$, body mass index; $\mathrm{AFC}$, antral follicle count.

a) Adjusted for embryo age, serum AMH level, AFC, and transferred embryo quality with embryo age and transferred embryo quality remaining independently significant.

prognosis with or without $\mathrm{GH}$ treatment (i.e., [-]GH or [+]GH). However, of the poor-prognosis patients, those receiving GH were signif- cantly older than those who did not, which reflected the transition to GH use if earlier cycles failed (Table 1). This trend was repeated pre- 
Table 3. Overview of patient characteristics and clinical outcomes in poor-prognosis-matched cohort with or without GH

\begin{tabular}{lccc}
\hline \multirow{2}{*}{ Variable } & \multicolumn{2}{c}{ Group matched for cycle age } & p-value \\
\cline { 2 - 3 } & $(-) \mathrm{GH}$ & $(+) \mathrm{GH}$ & - \\
\hline No. of cycles & 85 & 85 & - \\
Cycle age $(\mathrm{yr})$ & $38.0 \pm 4.0$ & $38.0 \pm 4.0$ & $0.814^{\mathrm{b})}$ \\
Embryo age $(\mathrm{yr})$ & $37.0 \pm 4.0$ & $37.0 \pm 4.0$ & $0.756^{\mathrm{c}}$ \\
AMH $(\mathrm{pmol} / \mathrm{L})^{\mathrm{a})}$ & $9.2(18.6)$ & $4.6(12.3)$ & $0.082^{\mathrm{c}}$ \\
BMI $\left(\mathrm{kg} / \mathrm{m}^{2}\right)^{\mathrm{a})}$ & $24.2(8.5)$ & $22.5(5.6)$ & - \\
FET cycle & 85 & 85 & $0.203^{\mathrm{d})}$ \\
FET pregnancy rate & $23 / 85(27.1)$ & $29 / 85(34.1)$ & $0.072^{\mathrm{d})}$ \\
FET live birth rate & $15 / 85(17.6)$ & $24 / 85(28.2)$ & $0.130^{\mathrm{d})}$ \\
FET miscarriage rate & $8 / 23(34.8)$ & $5 / 29(17.2)$ & \\
\hline
\end{tabular}

Values are presented as mean \pm standard deviation or number (\%) unless otherwise indicated.

$\mathrm{GH}$, growth hormone; $\mathrm{AMH}$, anti-Müllerian hormone; BMI, body mass index; FET, frozen embryo transfer.

${ }^{\text {a) }}$ Median (interquartile range); ${ }^{\text {b) }}$ Statistically significantly different from the $(-) \mathrm{GH}$ group; ${ }^{\mathrm{c})}$ Student $t$-test; ${ }^{\mathrm{d})}$ Kruskal-Wallis test; ${ }^{\text {e) }} \mathrm{Chi}$-square test.

cisely across the treatment groups for average embryo age, albeit those ages were approximately $7-10$ months younger than the cycle age for each treatment group, reflecting previous cryopreservation (Table 1). As expected, the median AMH level was significantly higher in the (-)Adj (Good) group (18.8 pmol/L) than in the poor-prognosis groups, (-)GH and (+) GH (9.2 and $4.6 \mathrm{pmol} / \mathrm{L}$, respectively). However, these latter AMH values were not significantly different in comparison to each other (Table 1).

The highest CP and LB rates were observed in the (-)Adj (Good) group, as expected, and lower rates were observed in the poor-prognosis groups ([-]GH and [+]GH). There were no significant differences between these latter groups in the CP or LB rates using chi-square analysis, and no significant difference was observed in the miscarriage rate across all treatment groups (Table 1). These observations for $\mathrm{CP}$ were confirmed using binary logistic regression in univariate and multivariate analyses adjusting for cycle or embryo age, $\mathrm{AMH}$, AFC and transferred embryo quality (Table 2). However, when adjusting for the same parameters in a multivariate logistic regression analysis of the likelihood of $L B$, the (-)GH group was significantly less likely to achieve a $\mathrm{LB}(\mathrm{OR}, 0.38 ; 95 \% \mathrm{Cl}, 0.21-0.70 ; p=0.002)$ than the (-) Adj (Good) group, while the likelihood of LB in the (+)GH group was not significantly different from that in the (-)Adj (Good) group (OR, $0.80 ; 95 \% \mathrm{Cl}, 0.39-1.65 ; p=0.551$ ) (Table 2). The other significant univariate predictors of $\mathrm{CP}$ and $\mathrm{LB}$ were cycle/embryo age, serum $\mathrm{AMH}$, AFC rating, and transferred embryo quality (Table 2). However, only transferred embryo quality and cycle or embryo age were retained in the multivariate logistic regression model (Table 2). RIF was also examined as a confounder, although only 38 patients in the entire cohort had experienced three or more implantation failures. While RIF was associated with a lower likelihood of $\mathrm{CP}(\mathrm{OR}, 0.28$; $95 \% \mathrm{Cl}, 0.12-$ $0.64 ; p=0.003)$ and $\mathrm{LB}(\mathrm{OR}, 0.23 ; 95 \% \mathrm{Cl}, 0.08-0.066 ; p=0.006)$ in univariate analyses, it was not significant in the multivariate models
(CP: OR, 0.54; 95\% Cl, 0.16-1.84; $p=0.325$ and LB: OR, $0.16 ; 95 \% \mathrm{Cl}$ $0.02-1.32 ; p=0.089$ ) (data not shown).

\section{Analysis of the cycle age-matched (-)GH versus (+)GH poor- prognosis groups}

To further explore the difference in outcomes between the two poor-prognosis groups (-) GH and (+) GH, a 1 to 1 randomized matched analysis was performed using cycle age as the connecting criterion (Table 3). This generated 170 cases with 85 cycles/women in each of the (-)GH and (+)GH groups. There were no significant differences in embryo age, AMH, BMl or clinical outcomes including CP, LB and miscarriage rates (Table 3). Precisely the same pattern that was observed in the whole cohort analysis was also observed when applying binary logistic regression for the likelihood of $C P$ and $L B$ in the cycle age-matched analyses (Table 4). The univariate analysis showed that increased cycle/embryo age was associated with a reduced likelihood of CP and LB, while a higher AFC grade and transferred embryo quality were associated with a significantly increased chance of these outcomes (Table 4). The presence of GH was not correlated with changes in the $\mathrm{OR}$ in the univariate analysis in comparison to $(-) \mathrm{GH}$ (Table 4). However, in a multivariate model adjusting for embryo age, AFC grade and transferred embryo quality, the (+)GH group showed an increased chance of $L B$ in comparison to the (-) GH group (OR, 2.71; $95 \% \mathrm{Cl}, 1.14-6.46 ; p=0.024$ ) (Table 4), but had no effect on the likelihood of CP. The same tendency was found if cycle age was factored into the analysis instead of embryo age (data not shown). While advancing cycle age and embryo age were again associated with a reduced chance of both clinical outcomes, women with an AFC of fewer than eight follicles (group D/E) had a lower likelihood of CP and LB (Table 4). This was demonstrated only for $L B$ following adjustment for transferred embryo quality, GH use and either cycle age or embryo age (Table 4). Importantly, after adjustment for cycle or embryo age, 
Table 4. Binary logistic regression analysis of poor-prognosis-matched cohort with or without GH

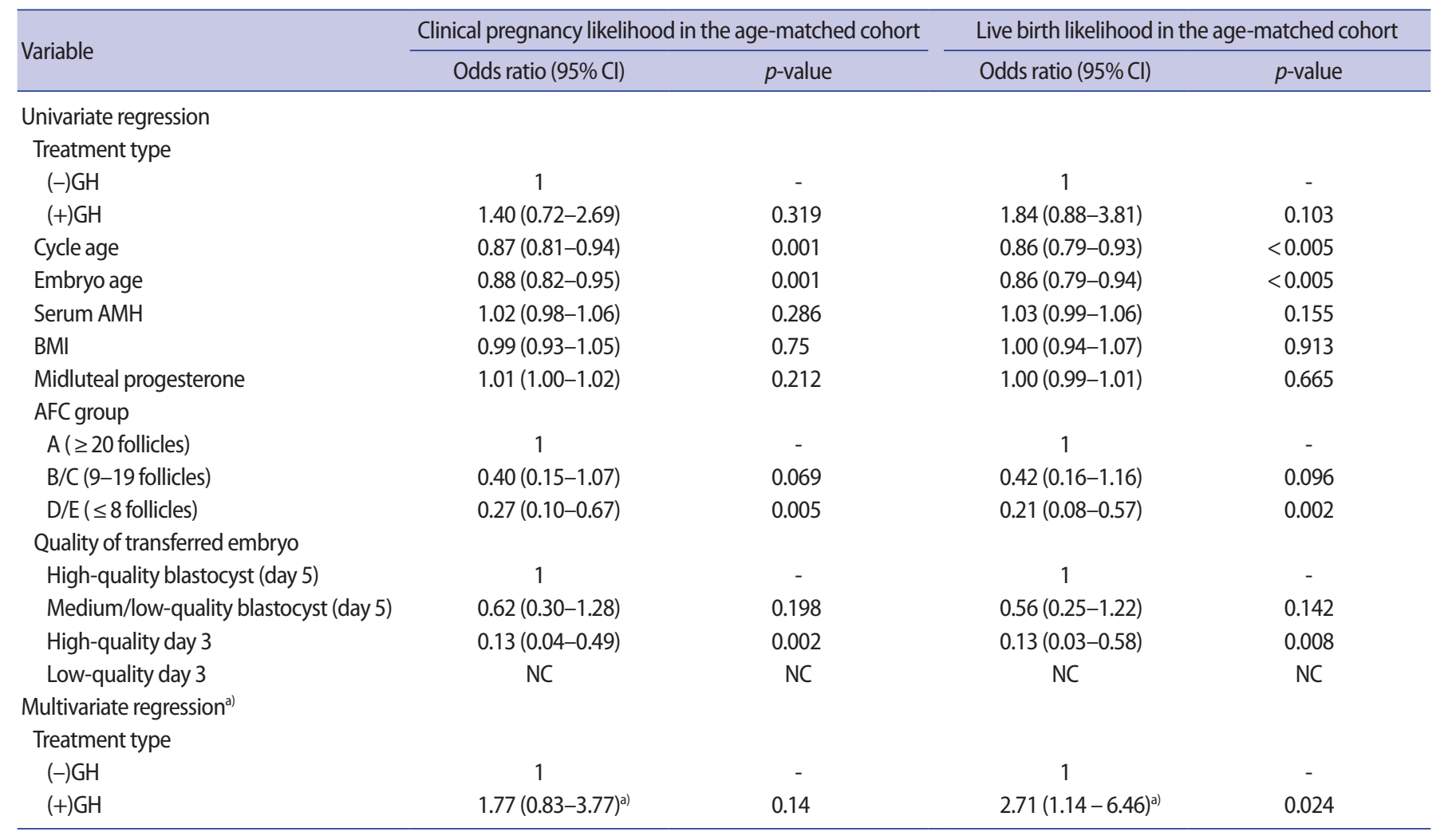

$\mathrm{GH}$, growth hormone; $\mathrm{Cl}$, confidence interval; $\mathrm{AMH}$, anti-Müllerian hormone; BMI, body mass index; $\mathrm{AFC}$, antral follicle count; $\mathrm{NC}$, not computed as the case number was too low.

${ }^{\text {a) }}$ Adjusted for embryo age, serum AMH level, AFC, and transferred embryo quality with embryo age and transferred embryo quality remaining independently significant.

AFC grade and transferred embryo quality, the use of GH in poorprognosis women led to a 2.7-fold increased chance of LB (Table 4).

\section{Analysis of the cycle age-matched good-prognosis (-)Adj (Good) group versus the poor-prognosis (+)GH group}

Finally, the effect of $\mathrm{GH}$ in poor-prognosis patients was compared to the outcomes of patients with a good prognosis ([-]Adj [Good]) in a matched analysis to reveal whether $\mathrm{GH}$ restored clinical outcomes to the level that would be expected in younger and more fertile women. The same 1 to 1 randomized matched approach was performed using cycle age as the connecting criterion (Supplementary Table 1) and showed that no outcome parameter was significantly different between the groups, with the exception of serum $\mathrm{AMH}$, which was significantly higher in the (-)Adj (Good) group, as expected. In the univariate analysis, the same outcomes were associated with an altered likelihood of CP and LB, notably cycle and embryo age, AFC grade and transferred embryo quality (Supplementary Table 2). In the multivariate analysis, only transferred embryo quality and cycle/embryo age were linked to a changed OR for CP (Supplementary Table 2). AFC was also a predictor of the likelihood of CP and
LB in the univariate model (Supplementary Table 2), but the association disappeared when adjusting for cycle age (data not shown), embryo age or transferred embryo quality (Supplementary Table 2). Importantly, in both models using either cycle or embryo age, there was no significant difference between the two treatment groups (i.e., [-]Adj [Good] and [+]GH), for the likelihood of either CP or LB, indicating that in an age-matched analysis, GH led to a similar CP and LB likelihood, which was similar to that of patients with a better clinical prognosis (Supplementary Table 2).

\section{Discussion}

The cohort of any ART clinic can be divided into two distinct groups: those with a poor prognosis and those with a good prognosis. This distinction is based on the likelihood of achieving $\mathrm{CP}$ and/or $L B$, and incorporates key determinants of fertility. Female age is the single factor most definitively associated with the CP and LB rate [26], and is also indicative of a woman's ovarian reserve of primordial follicles remaining in the ovary. The AFC and the AMH level are measures that predict ovarian reserve and underpin the prescription dosage of 
exogenous gonadotrophins that are replaced after hypothalamic gonadotrophin-releasing hormone suppression in IVF stimulation [2729]. The categorization of women as poor-prognosis is generally accomplished by applying the Bologna criteria, which state that poorprognosis women meet two of the following three parameters: advanced maternal age ( $\geq 40$ years), low ovarian reserve (AMH $\leq 3.6-$ $7.9 \mathrm{pmol} / \mathrm{L}$ or AFC $<5-7$ follicles), or poor ovarian response with conventional stimulation ( $\leq 3$ oocytes retrieved) [30]. However, the applicability of these criteria is debated and researchers are developing more nuanced approaches, such as Patient-Oriented Strategies Encompassing IndividualizeD Oocyte Number (POSEIDON), to define "low"-prognosis patients [31]. Nonetheless, these classifications have not been able to define subsets of poor-prognosis patients who can benefit from various ART interventions including adjuvant co-treatment [31]. In our previous studies, we introduced the concept of reduced embryo quality as an additional diagnostic parameter for poor-prognosis patients, whereby those with $>50 \%$ of embryos with marked fragmentation are predicted to have poorer outcomes $[1,12,13]$. In the current study, we used the same criteria to define a poor prognosis, and it is these patients who are offered adjuvant therapies such as GH in order to enhance their primary ART outcomes.

We reported that after adjustment for critical variables such as female cycle age, embryo age, AFC and transferred embryo quality, the $\mathrm{CP}$ and $\mathrm{LB}$ rates in the $\mathrm{GH}$-treated group $([+] \mathrm{GH})$ were lower but not statistically significantly different from the younger, good-prognosis patients who did not receive adjuvant therapy ([-]Adj [Good]). Furthermore, the likelihood of CP and LB was lowest in the group of poor-prognosis women who did not receive $\mathrm{GH}([-] \mathrm{GH})$, which was maintained following adjustment for the same covariates. Interestingly, in the cycle age-matched analysis, the $(+) \mathrm{GH}$ group showed similar outcomes to those of the good-prognosis group, (-)Adj (Good), and significantly better outcomes than those of the (-) $\mathrm{GH}$ group. In this comparison, the (+)GH group showed a up to 2-fold increase in the likelihood of LB in the multivariate analysis, following adjustment for age, AFC and transferred embryo quality. The increased likelihood of LB in embryos previously generated under the action of GH was supported by a corresponding reduction in the miscarriage rate; this reduction was not significant, but may provide support for the premise that GH cotreatment improves oocyte maturation during folliculogenesis in the preceding stimulated cycle $[32,33]$. This trend for $\mathrm{GH}$ was also evident in our previous studies of fresh ET generated under the action of $\mathrm{GH}[12,13]$.

In accordance with this previous work on fresh transfers [12,13], the current findings indicated that the use of $\mathrm{GH}$ as an adjuvant cotreatment during IVF stimulation had a positive outcome on fresh ET and in addition, in subsequent FET cycles. As GH was not administered during the FET cycles, this suggested that the potential beneficial effects of GH may extend to embryo quality as presented previously $[5,9,16]$, rather than involving an effect on endometrium receptivity $[15,17]$. However, in our previous studies on embryos generated under $\mathrm{GH}$ action, we failed to demonstrate that the adjuvant altered embryo quality to a measurable extent, as the proportion of highquality embryos on morphological assessment was the same with or without GH $[12,13]$. This echoes the observations of the LIGHT study, where no significant increase in measurable embryo quality was detected [8]. However, they showed that patients receiving $\mathrm{GH}$ reached oocyte retrieval more rapidly than those who did not receive adjuvant therapy, which may indicate a potential effect on folliculogenesis [8]. Furthermore, in our previous reports, patients receiving $\mathrm{GH}$ had higher oocyte and embryo utilization rates, which indicated that more "usable" embryos were either transferred or cryopreserved. While this is not a direct measurement of embryo quality, it does imply that those with GH may have more opportunities for ET (and ensuing pregnancies) in later cycles. Nonetheless, several other reports have demonstrated that $\mathrm{GH}$ enhanced embryo quality as assessed by morphological grading $[5,16]$, although in our study this enhancement was not detectable. It is possible that such embryos (and the oocytes from which they were derived) have higher competency for generating pregnancies, which is not measured by current morphological grading systems.

Other investigations have found that $\mathrm{GH}$ cotreatment increased CP and LB rates for poor-prognosis patients by $10 \%-20 \%[1,2,12]$. A retrospective analysis of younger poor responders and patients with previous unsuccessful IVF attempts estimated that GH increased CP rates by $25 \%-30 \%$ [34], but had little effect on those with advanced maternal age. Our own recent retrospective analysis also showed little benefit in women over 41 years of age [12], which has implications for $\mathrm{GH}$ use in poor-prognosis patients with at least one Bologna criterion, advanced age [30]. However, in prospective studies with 80-145 participants, other researchers have shown that GH had no influence on CP or LB rates in ART $[3,4,6]$. Moreover, systematic reviews of the literature are also divided on the therapeutic effects of $\mathrm{GH}$, possibly because of the heterogeneity among clinical studies. Two recent meta-analyses indicated that $\mathrm{GH}$ had no significant influence on the LB rate $[35,36]$, but yielded a slight increase in the pregnancy rate [35]. Several other systematic reviews and meta-analyses have indicated that $\mathrm{GH}$ co-treatment significantly increased the $\mathrm{OR}$ for CP from 1.6 to 3.3, and the OR for LB from 1.7 to 5.4 [37-40]. Previously, we demonstrated that GH independently increased the likelihood of CP in fresh transfers with an approximate OR of $2.5(95 \% \mathrm{Cl}$, 1.42-4.37) and increased the likelihood of LB, with an OR of $4.8(95 \%$ $\mathrm{Cl}, 2.30-9.79)$ [13]. These ORs are in line with the studies outlined above and are also in accordance with the observations of the cur- 
rent FET study, in which GH independently increased the chances of LB by about 2.7-fold.

Importantly, very few studies have investigated the role of GH cotreatment either directly in FET cycle set-ups or in subsequent FET cycles derived from $\mathrm{GH}$ used in stimulated cycles. Two recent reports suggested that direct GH co-treatment during the HRT phase of FET cycles led to increased endometrium thickness, with subsequent increases in the implantation and CP rates [14,15], and increased LB rates in a prospective study with 230 participants [14]. Furthermore, in an RCT with freshly transferred donor embryos, Altmae et al. [17] showed that donor recipients who received GH as part of their HRT regimen had greater endometrium thickness, along with higher pregnancy and LB rates. Cui et al. [15] also performed in vitro experiments and showed that GH upregulated insulin-like growth factor 1, along with the proangiogenic factor vascular endothelial growth factor in an endometrial carcinoma cell line, which may modify endometrial receptivity [41] and aid decidualization. The potential effect of $\mathrm{GH}$ on endometrial receptivity is intriguing, but requires further research, as the more traditional hypothesis has centered around improved embryo quality [9].

Given the administrative and financial resources required, the LIGHT investigators were unable to examine the subsequent FET outcomes from the stimulated cycles with GH therapy in their RCT [8]. This could have yielded insights on how these embryos performed later and provided a reflection of embryo quality beyond standard morphology. However, our group examined this issue in a retrospective fashion in 2010 [1], and now in 2019, in two separate cohorts from both studies we have demonstrated positive effects in subsequent FET cycles that were conducted without the influence of exogenous GH. These data may indicate a potential "carry-over" effect from GH used during stimulation, which is likely to be mediated by undetectable changes in embryo quality, suggesting that the potential beneficial mechanism of $\mathrm{GH}$ for clinical outcomes is more likely to be related to embryo quality than endometrial receptivity.

Unfortunately, like all retrospective analyses, these studies have significant limitations, which should be cautiously considered when interpreting the findings. The potential influential effects of $\mathrm{GH}$ are strictly associative rather than causative, as this study was not designed as an interventional RCT. Furthermore, there was significant heterogeneity in terms of the factors linked to a poor prognosis factors and the combination thereof in the adjuvant treatment groups. These groups tended to have a higher proportion of patients with multiple factors linked to a poor prognosis, and this heterogeneity is a significant limitation. In addition, the definition of a poor prognosis is not precisely in line with international criteria, weakening the study design, but we propose that the generation of embryos of reduced quality should be directly consider as a criterion for poor prognosis.
Prospective studies have the advantage of focusing on homogenous populations with well-defined criteria of a poor prognosis. However, as discussed here, the current criteria are a matter of debate; future studies could incorporate factors outside of the traditional Bologna criteria and possibly take a more nuanced approach like that of the POSEIDON study [31]. In addition, while our study design attempted to minimize patient selection bias, its retrospective nature means that this could not be completely averted. The selection of the (-)GH control group meant that this group was composed of patients who were offered adjuvant therapy due to one or more of the five reasons outlined in the Methods section, but their first chronological cycle within the study period did not include an adjuvant. This reflected our attempt to select a poor-prognosis cohort, but to randomly choose cycles without adjuvant intervention. The secondary aim of this process was to prevent any confounding that could result from including multiple treatment cycles in individual women. Other potential factors that were not examined included patient's socioeconomic status and parity. Affordability could be a critical confounder, as patients who used adjuvants in the stimulated cycle were required to pay for the rather expensive $\mathrm{GH}$.

Conversely, there are significant strengths associated with the current study including the use of a large data-set ( $n=1,119$ cycles) with a specific and well-established HRT regimen, along with a focus on the transfer of single autologous embryos that had been vitrified using the same technique. In addition, we analysed only the first chronological FET cycle for each patient within the study time frame, as a mechanism to minimize patient selection bias. The study was also comprehensive in examining the independent influence of several potential confounding variables such as age, AFC, AMH level, transferred embryo quality, endometrial thickness, $E_{2}$ and $P 4$ levels, which were also used as covariates in multiple regression analyses (data not shown).

Taken together, while not as robustly designed and as powerful as a prospective RCT, the current study design limited any perceived bias, and is one of the largest studies of GH in FET cycles to date. It is increasingly difficult to recruit ART patients into well-designed RCTs, as demonstrated by the LIGHT study [8]. This is an issue for all ART studies, as patient demand in commercial clinics overpowers the ability to recruit, leaving researchers in the field with vastly underpowered studies. If this cannot be addressed, progress may only be achieved by useful retrospective studies that are stringently designed, conducted and analysed. Nonetheless, the current investigation provided further evidence to suggest that $\mathrm{GH}$ administration in stimulation cycles may potentially cause changes in developing oocytes that lead to better LB rates when the embryos derived from these oocytes are transferred later in frozen cycles. This potential change may be reflected by enhanced embryo quality that is not de- 
tectable by conventional morphological assessments, but provides improved competency for the generation of pregnancies.

\section{Conflict of interest}

No potential conflict of interest relevant to this article was reported.

\section{Acknowledgments}

All patients and staff at PIVET Medical Center are acknowledged with thanks.

\section{ORCID}

Kevin N Keane

https://orcid.org/0000-0001-6248-7705

Satvinder S Dhaliwal

https://orcid.org/0000-0002-4476-1042

John LYovich

https://orcid.org/0000-0002-9583-3683

\section{Author contributions}

Conceptualization: KNK, SSD, JLY. Data curation: KNK, PMH. Formal analysis: KNK, YY, SLPR, SSD. Funding acquisition: KNK, JLY. Methodology: KNK, YY, SSD, JLY. Project administration: KNK, JLY. Visualization: KNK. Writing - original draft: JLY, KNK. Writing - review \& editing: all authors.

\section{Supplementary materials}

Supplementary materials can be found via https://doi.org/10.5653/ cerm.2019.00206.

\section{References}

1. Yovich JL, Stanger JD. Growth hormone supplementation improves implantation and pregnancy productivity rates for poorprognosis patients undertaking IVF. Reprod Biomed Online 2010;21:37-49.

2. Tesarik J, Hazout A, Mendoza C. Improvement of delivery and live birth rates after ICSI in women aged $>40$ years by ovarian costimulation with growth hormone. Hum Reprod 2005;20:253641.

3. Bassiouny YA, Dakhly DM, Bayoumi YA, Hashish NM. Does the addition of growth hormone to the in vitro fertilization/intracytoplasmic sperm injection antagonist protocol improve outcomes in poor responders? A randomized, controlled trial. Fertil Steril 2016;105:697-702.

4. Bayoumi YA, Dakhly DM, Bassiouny YA, Hashish NM. Addition of growth hormone to the microflare stimulation protocol among women with poor ovarian response. Int J Gynaecol Obstet 2015;131:305-8.

5. Lattes K, Brassesco M, Gomez M, Checa MA. Low-dose growth hormone supplementation increases clinical pregnancy rate in poor responders undergoing in vitro fertilisation. Gynecol Endocrinol 2015;31:565-8.

6. Eftekhar M, Aflatoonian A, Mohammadian F, Eftekhar T. Adjuvant growth hormone therapy in antagonist protocol in poor responders undergoing assisted reproductive technology. Arch Gynecol Obstet 2013;287:1017-21.

7. Haydardedeoglu B, Isik AZ, Kilicdag EB. The combination of dhea, transdermal testosterone and growth hormone as an adjuvant therapy in assisted reproductive technology in patients with dor below 40 years of age. Fertil Steril 2014;102:e221-2.

8. Norman RJ, Alvino H, Hull LM, Mol BW, Hart RJ, Kelly TL, et al. Human growth hormone for poor responders: a randomized placebo-controlled trial provides no evidence for improved live birth rate. Reprod Biomed Online 2019;38:908-15.

9. Ob'edkova K, Kogan I, Krikheli I, Dzhemlikhanova L, Muller V, Mekina I, et al. Growth hormone co-treatment in IVF/ICSI cycles in poor responders. Gynecol Endocrinol 2017;33(sup1):15-7.

10. Norman RJ, Alvino H, Hart R, Rombauts L. A randomised double blind placebo controlled study of recombinant human growth hormone (r-HGH) on live birth rates in women who are poor responders. Hum Reprod 2016;31(Suppl 1):i37.

11. Bevers MM, Izadyar F. Role of growth hormone and growth hormone receptor in oocyte maturation. Mol Cell Endocrinol 2002; 197:173-8.

12. Keane KN, Yovich JL, Hamidi A, Hinchliffe PM, Dhaliwal SS. Single-centre retrospective analysis of growth hormone supplementation in IVF patients classified as poor-prognosis. BMJ Open 2017;7:e018107.

13. Keane KN, Hinchliffe PM, Rowlands PK, Borude G, Srinivasan S, Dhaliwal SS, et al. DHEA supplementation confers no additional benefit to that of growth hormone on pregnancy and live birth rates in IVF patients categorized as poor prognosis. Front Endocrinol (Lausanne) 2018;9:14.

14. Xue-Mei W, Hong J, Wen-Xiang Z, Yang L. The effects of growth hormone on clinical outcomes after frozen-thawed embryo transfer. Int J Gynaecol Obstet 2016;133:347-50.

15. Cui N, Li AM, Luo ZY, Zhao ZM, Xu YM, Zhang J, et al. Effects of growth hormone on pregnancy rates of patients with thin endometrium. J Endocrinol Invest 2019;42:27-35.

16. Chu K, Pang W, Sun N, Zhang Q, Li W. Outcomes of poor responders following growth hormone co-treatment with IVF/ICSI mild stimulation protocol: a retrospective cohort study. Arch Gy- 
necol Obstet 2018;297:1317-21.

17. Altmae S, Mendoza-Tesarik R, Mendoza C, Mendoza N, Cucinelli $\mathrm{F}$, Tesarik J. Effect of growth hormone on uterine receptivity in women with repeated implantation failure in an oocyte donation program: a randomized controlled trial. J Endocr Soc 2017; 2:96-105.

18. Kuwayama M, Vajta G, Kato O, Leibo SP. Highly efficient vitrification method for cryopreservation of human oocytes. Reprod Biomed Online 2005;11:300-8.

19. Yovich J, Stanger J, Hinchliffe P. Targeted gonadotrophin stimulation using the PIVET algorithm markedly reduces the risk of OHSS. Reprod Biomed Online 2012;24:281-92.

20. Yovich JL, Alsbjerg B, Conceicao JL, Hinchliffe PM, Keane KN. PIVET rFSH dosing algorithms for individualized controlled ovarian stimulation enables optimized pregnancy productivity rates and avoidance of ovarian hyperstimulation syndrome. Drug Des Devel Ther 2016;10:2561-73.

21. Keane KN, Mustafa KB, Hinchliffe P, Conceicao J, Yovich JL. Higher $\beta$-HCG concentrations and higher birthweights ensue from single vitrified embryo transfers. Reprod Biomed Online 2016;33: 149-60.

22. Yovich JL, Conceicao JL, Stanger JD, Hinchliffe PM, Keane KN. Mid-luteal serum progesterone concentrations govern implantation rates for cryopreserved embryo transfers conducted under hormone replacement. Reprod Biomed Online 2015;31:180-91.

23. Yovich JL, Conceicao J, Hinchliffe P, Keane K. Which blastocysts should be considered for genetic screening? Hum Reprod 2015;30:1743-4.

24. Gardner DK, Schoolcraft WB. In vitro culture of human blastocyst. In: Jansen R, Mortimer D, editors. Towards reproductive certainty: fertility and genetics beyond 1999. Carnforth: Parthenon; 1999. p. 378-88.

25. Yovich JL, Stanger JD, Yovich JM, Tuvik Al. Quality of embryos from in-vitro fertilisation. Lancet 1984;1:457.

26. Rosen MP, Johnstone E, Addauan-Andersen C, Cedars MI. A lower antral follicle count is associated with infertility. Fertil Steril 2011;95:1950-4.

27. Almog B, Shehata F, Shalom-Paz E, Tan SL, Tulandi T. Age-related normogram for antral follicle count: McGill reference guide. Fertil Steril 2011;95:663-6.

28. van Rooij IA, Broekmans FJ, Scheffer GJ, Looman CW, Habbema JD, de Jong FH, et al. Serum antimullerian hormone levels best reflect the reproductive decline with age in normal women with proven fertility: a longitudinal study. Fertil Steril 2005;83:979-87.

29. Keane K, Cruzat VF, Wagle S, Chaudhary N, Newsholme P, Yovich J. Specific ranges of anti-Mullerian hormone and antral follicle count correlate to provide a prognostic indicator for IVF out- come. Reprod Biol 2017; 17:51-9.

30. Ferraretti AP, Gianaroli L. The Bologna criteria for the definition of poor ovarian responders: is there a need for revision? Hum Reprod 2014;29:1842-5.

31. Humaidan P, Alviggi C, Fischer R, Esteves SC. The novel POSEIDON stratification of 'low prognosis patients in Assisted Reproductive Technology' and its proposed marker of successful outcome. F1000Res 2016;5:2911.

32. Izadyar F, Colenbrander B, Bevers MM. Stimulatory effect of growth hormone on in vitro maturation of bovine oocytes is exerted through the cyclic adenosine 3',5'-monophosphate signaling pathway. Biol Reprod 1997;57:1484-9.

33. Regan SL, Knight PG, Yovich JL, Arfuso F, Dharmarajan A. Growth hormone during in vitro fertilization in older women modulates the density of receptors in granulosa cells, with improved pregnancy outcomes. Fertil Steril 2018;110:1298-310.

34. Ho YK, Lee TH, Lee Cl, Cheng EH, Huang CC, Huang LS, et al. Effects of growth hormone plus gonadotropins on controlled ovarian stimulation in infertile women of advanced age, poor responders, and previous in vitro fertilization failure patients. Taiwan J Obstet Gynecol 2017;56:806-10.

35. Hart RJ, Rombauts L, Norman RJ. Growth hormone in IVF cycles: any hope? Curr Opin Obstet Gynecol 2017;29:119-25.

36. Yu X, Ruan J, He LP, Hu W, Xu Q, Tang J, et al. Efficacy of growth hormone supplementation with gonadotrophins in vitro fertilization for poor ovarian responders: an updated meta-analysis. Int J Clin Exp Med 2015;8:4954-67.

37. Li XL, Wang L, Lv F, Huang XM, Wang LP, Pan Y, et al. The influence of different growth hormone addition protocols to poor ovarian responders on clinical outcomes in controlled ovary stimulation cycles: a systematic review and meta-analysis. Medicine (Baltimore) 2017;96:e6443.

38. Duffy JM, Ahmad G, Mohiyiddeen L, Nardo LG, Watson A. Growth hormone for in vitro fertilization. Cochrane Database Syst Rev 2010;(1):CD000099.

39. Kyrou D, Kolibianakis EM, Venetis CA, Papanikolaou EG, Bontis J, Tarlatzis BC. How to improve the probability of pregnancy in poor responders undergoing in vitro fertilization: a systematic review and meta-analysis. Fertil Steril 2009;91:749-66.

40. Jeve YB, Bhandari HM. Effective treatment protocol for poor ovarian response: a systematic review and meta-analysis. J Hum Reprod Sci 2016;9:70-81.

41. Horcajadas JA, Riesewijk A, Polman J, van Os R, Pellicer A, Mosselman $S$, et al. Effect of controlled ovarian hyperstimulation in IVF on endometrial gene expression profiles. Mol Hum Reprod 2005;11:195-205. 
Supplementary Table 1. Overview of cycle age-matched analysis of good-prognosis patients versus poor-prognosis patients treated with GH

\begin{tabular}{|c|c|c|c|}
\hline \multirow{2}{*}{ Variable } & \multicolumn{2}{|c|}{ Matched for cycle age } & \multirow{2}{*}{$p$-value } \\
\hline & (-)Adj (Good) & $(+) \mathrm{GH}$ & \\
\hline No. of cycles & 89 & 89 & - \\
\hline Embryo age (yr) & $37.2 \pm 4.5$ & $37.7 \pm 4.5$ & $0.473^{c)}$ \\
\hline $\mathrm{AMH}(\mathrm{pmol} / \mathrm{L})^{\mathrm{a})}$ & $18.8(29.1)$ & $4.6(12.3)^{b)}$ & $0.012^{\mathrm{d})}$ \\
\hline BMI $\left(\mathrm{kg} / \mathrm{m}^{2}\right)^{\mathrm{a})}$ & $23.3(6.5)$ & $22.5(5.6)$ & $0.589^{d)}$ \\
\hline FET pregnancy rate & $36 / 89(40.4)$ & $27 / 89(30.3)$ & $0.105^{\mathrm{e})}$ \\
\hline FET live birth rate & $24 / 89(27.0)$ & $22 / 89(24.7)$ & $0.432^{\mathrm{e})}$ \\
\hline FET miscarriage rate & $12 / 36(33.3)$ & $5 / 27(18.5)$ & $0.153^{\mathrm{e})}$ \\
\hline
\end{tabular}

Values are presented as mean \pm standard deviation or number (\%) unless otherwise indicated.

$\mathrm{GH}$, growth hormone; $\mathrm{AMH}$, anti-Müllerian hormone; BMI, body mass index; FET, frozen embryo transfer.

${ }^{a}$ ) Median (interquartile range); ${ }^{\text {b)} S t a t i s t i c a l l y ~ s i g n i f i c a n t l y ~ d i f f e r e n t ~ f r o m ~ t h e ~(-) A d j ~(G o o d) ~ g r o u p ; ~}{ }^{\text {c }}$ Student $t$-test; ${ }^{\text {d) }}$ Kruskal-Wallis test; ${ }^{\text {e)}}$ Chi-square test. 
Supplementary Table 2. Complete univariate and multivariate analysis of embryo age-matched good-prognosis patients versus poor-prognosis patients treated with GH

\begin{tabular}{|c|c|c|c|c|}
\hline \multirow{2}{*}{ Variable } & \multicolumn{2}{|c|}{ Clinical pregnancy likelihood in the age-matched cohort } & \multicolumn{2}{|c|}{ Live birth likelihood in the age-matched cohort } \\
\hline & Odds ratio $(95 \% \mathrm{Cl})$ & $p$-value & Odds ratio $(95 \% \mathrm{Cl})$ & $p$-value \\
\hline \multicolumn{5}{|l|}{ Univariate regression } \\
\hline \multicolumn{5}{|l|}{ Treatment type } \\
\hline$(-) \mathrm{GH}$ & 1 & - & 1 & - \\
\hline$(+) \mathrm{GH}$ & $0.64(0.34-1.19)$ & 0.159 & $0.89(0.45-1.74)$ & 0.732 \\
\hline Cycle age & $0.89(0.83-0.96)$ & 0.001 & $0.89(0.82-0.96)$ & 0.002 \\
\hline Embryo age & $0.88(0.82-0.95)$ & 0.001 & $0.88(0.81-0.95)$ & 0.001 \\
\hline Serum AMH & $1.01(0.97-1.04)$ & 0.680 & $1.02(0.98-1.06)$ & 0.268 \\
\hline BMl & $0.98(0.92-1.03)$ & 0.406 & $0.97(0.92-1.04)$ & 0.418 \\
\hline \multicolumn{5}{|l|}{ AFC group } \\
\hline A ( $\geq 20$ follicles) & 1 & - & 1 & - \\
\hline B/C (9-19 follicles) & $0.47(0.20-1.12)$ & 0.089 & $0.61(0.25-1.50)$ & 0.282 \\
\hline $\mathrm{D} / \mathrm{E}$ ( $\leq 8$ follicles $)$ & $0.24(0.10-0.57)$ & 0.001 & $0.28(0.11-0.70)$ & 0.007 \\
\hline \multicolumn{5}{|l|}{ Quality of transferred embryo } \\
\hline High-quality blastocyst (day 5) & 1 & - & 1 & - \\
\hline Medium/low-quality blastocyst (day 5) & $0.49(0.24-0.98)$ & 0.043 & $0.35(0.16-0.78)$ & 0.01 \\
\hline High-quality day 3 & $0.22(0.07-0.69)$ & 0.01 & $0.23(0.06-0.84)$ & 0.026 \\
\hline Low-quality day 3 & NC & NC & $\mathrm{NC}$ & NC \\
\hline \multicolumn{5}{|l|}{ Multivariate regression ${ }^{\mathrm{a})}$} \\
\hline \multicolumn{5}{|l|}{ Treatment type } \\
\hline (-)Adj (Good) & 1 & - & 1 & - \\
\hline$(+) \mathrm{GH}$ & $1.08(0.52-2.27)^{\mathrm{a})}$ & 0.833 & $1.55(0.69-3.48)^{\mathrm{a})}$ & 0.292 \\
\hline
\end{tabular}

$\mathrm{GH}$, growth hormone; $\mathrm{Cl}$, confidence interval; $\mathrm{AMH}$, anti-Müllerian hormone; BMI, body mass index; $\mathrm{AFC}$, antral follicle count; $\mathrm{NC}$, not computed as the case number was too low.

${ }^{\text {a) }}$ Adjusted for embryo age, AFC, and transferred embryo quality, with embryo age and AFC remaining independently significant. 